\title{
Enucleation and Exenteration
}

\author{
Santosh G. Honavar and Raksha Rao
}

Enucleation and exenteration for the management of intraocular and orbital malignancies, respectively, was the standard treatment of care before the advent of radiotherapy and chemotherapy. Over the past few decades, enhanced knowledge on the biology of the tumors, advances in the histopathologic and cytological techniques, use of various diagnostic modalities for an early and a more precise diagnosis, implementation of protocol-based management, and use of multimodal treatment including chemotherapy, radiotherapy, immunotherapy, and conservative surgeries, have greatly contributed to the reduction in the need for enucleation and exenteration. This paradigm shift toward less radical treatment has brought about a refinement in the absolute indications for enucleation and exenteration.

Electronic supplementary material The online version of this chapter (https://doi.org/10.1007/978-3-030-187576_12) contains supplementary material, which is available to authorized users.

S. G. Honavar $(\bowtie)$

Ocular Oncology Service and National

Retinoblastoma Foundation, Centre for Sight,

Hyderabad, India

R. Rao

Department of Orbit and Ophthalmic Oncology,

Narayana Nethralaya, Bangalore, India

\section{Overview and Indications of Enucleation}

Enucleation is defined as the removal of the eyeball while preserving the rest of the orbital tissues, including the extraocular muscles. Following enucleation, the aim is to achieve a good cosmesis by placing a prosthesis which matches the contralateral eye. A well-planned surgical technique with adequate volume replacement provides gratifying static and dynamic cosmesis. The characteristics of an ideal anophthalmic socket include a centrally placed well-covered implant of adequate volume, a socket lined with healthy conjunctiva, deep fornices to help retain the prosthesis, normal eyelid position with symmetric lid crease, and good implant motility which is transmitted to the overlying prosthesis.

In oncology practice, enucleation is routinely performed for the management of malignant intraocular tumors with an aim for complete tumor control [1-12]. To achieve this end, it sometimes entails the removal of an additional and adequate length of the optic nerve, excision of any adherent conjunctiva and tenon's fascia to the underlying tumor, or sacrificing a part of the orbital tissue, and this is termed as extended enucleation. Over the past few decades, advances in chemotherapy and radiotherapy have led to eyesalvaging treatment options for intraocular tumors, and the indications for enucleation have thus become specific [9, 10, 13-15]. 
Enucleation continues to play a major role in the management of retinoblastoma and uveal melanoma (Table 12.1). In retinoblastoma, indications for primary enucleation include advanced unilateral retinoblastoma without salvageable vision (group $\mathrm{E}$ of international classification of retinoblastoma [ICRB]), unilateral retinoblastoma with diffuse anterior chamber involvement, unilateral retinoblastoma with total vitreous hemorrhage, unilateral retinoblastoma with accompanying neovascular glaucoma, and retinoblastoma with a phthisical eye [1-3]. Secondary enucleation is performed for recurrent retinal tumor not controlled with current treatment modalities, vitreous seeds or subretinal seeds with suboptimal response to chemotherapy and/or radiotherapy, eyes with opaque media (total hyphema or dense vitreous hemorrhage) after an initial failed response to chemotherapy and/or radiotherapy, and as a part of the treatment protocol in orbital retinoblastoma [2-4].
Enucleation for ciliochoroidal melanoma as a primary treatment is indicated in very large tumors ( $>18 \mathrm{~mm}$ in diameter and $>10 \mathrm{~mm}$ in thickness) which are not amenable to plaque radiotherapy (Table 12.1) [5, 6]. Choroidal melanomas with optic nerve invasion are also an indication for enucleation [7]. Enucleation may be necessary in recurrent posterior melanoma, which has not responded favorably to radiation treatment $[5,6]$. Extraocular extension of melanoma also warrants enucleation, although there has been a recent trend to manage such cases by plaque brachytherapy where the size of the extraocular extension is less than $3 \mathrm{~mm}[8,9]$. Ciliochoroidal melanomas presenting with dense vitreous hemorrhage, or as a painful blind eye due to extensive necrosis or secondary glaucoma, are other indications for enucleation $[5,6]$.

Enucleation in iris melanoma is generally reserved for tumors which have progressed to a size where surgery or radiotherapy is not feasible (Table 12.1) [10,11]. These include eyes where

Table 12.1 Indications for enucleation

\begin{tabular}{|c|c|c|}
\hline Tumor & Primary & Secondary \\
\hline Retinoblastoma & $\begin{array}{l}\text { Advanced unilateral retinoblastoma } \\
\text { without salvageable vision } \\
\text { Diffuse anterior chamber } \\
\text { involvement } \\
\text { Total vitreous hemorrhage } \\
\text { Total hyphema } \\
\text { Unilateral retinoblastoma with } \\
\text { accompanying neovascular } \\
\text { glaucoma } \\
\text { Retinoblastoma with a phthisical eye }\end{array}$ & $\begin{array}{l}\text { Recurrent retinal tumor, vitreous seeds or subretinal } \\
\text { seeds with suboptimal response to chemotherapy and/ } \\
\text { or radiotherapy } \\
\text { Eyes with opaque media (total hyphema or dense } \\
\text { vitreous hemorrhage) after an initial failed response to } \\
\text { chemotherapy and/or radiotherapy } \\
\text { As a part of the treatment protocol in orbital } \\
\text { retinoblastoma }\end{array}$ \\
\hline $\begin{array}{l}\text { Ciliochoroidal } \\
\text { melanoma }\end{array}$ & $\begin{array}{l}\text { Very large tumors ( }>18 \mathrm{~mm} \text { in } \\
\text { diameter and }>10 \mathrm{~mm} \text { in thickness) } \\
\text { Optic nerve invasion } \\
\text { Extraocular extension of melanoma } \\
\text { more than } 3 \mathrm{~mm} \text { in size } \\
\text { Dense vitreous hemorrhage } \\
\text { Painful blind eye secondary to } \\
\text { extensive necrosis or secondary } \\
\text { glaucoma }\end{array}$ & $\begin{array}{l}\text { Recurrent posterior melanoma, which has not } \\
\text { responded favorably to radiation }\end{array}$ \\
\hline Iris melanoma & $\begin{array}{l}\text { Tumor involving all iris quadrants } \\
\text { Eyes with uncontrolled secondary } \\
\text { glaucoma with angle seeding } \\
\text { Eye with large extrascleral extension }\end{array}$ & $\begin{array}{l}\text { Recurrent iris melanoma, which has not responded } \\
\text { favorably to radiation }\end{array}$ \\
\hline Others & $\begin{array}{l}\text { Large medulloepithelioma } \\
\text { Intraocular extension of conjunctival } \\
\text { squamous cell carcinoma } \\
\text { Blind painful eyes harboring large } \\
\text { benign or malignant intraocular } \\
\text { tumors }\end{array}$ & $\begin{array}{l}\text { Recurrent tumors, which has not responded favorably } \\
\text { to other conservative treatment }\end{array}$ \\
\hline
\end{tabular}


the tumor involves all iris quadrants, eyes with uncontrolled secondary glaucoma with angle seeding, or in the presence of extrascleral extension [10, 11]. In a long-term analysis of 144 patients with iris melanoma treated by plaque radiotherapy, Shields et al. noted that glaucoma was present at the initial visit in $40 \%$ of cases, and by Kaplan-Meier estimate at 7 years, enucleation was necessary in $12 \%$ [11].

Enucleation is sometimes necessary in other conditions including medulloepithelioma, intraocular extension of conjunctival squamous cell carcinoma, and blind painful eyes harboring large benign or malignant intraocular tumors (Table 12.1) $[7,12]$. In a two-institutional study of 41 eyes with medulloepithelioma, 26 eyes $(63 \%)$ required enucleation, and it was mainly indicated in larger tumors $(n=21)$, and in smaller tumors that failed to respond to conservative treatment $(n=5)$ [12].

\section{Overview and Indications of Exenteration}

Orbital exenteration is the surgical removal of the orbital contents, including the eyeball. Exenteration is performed as a life-saving measure for primary or secondary orbital malignancies where conservative treatment has failed or is not feasible. Exenteration maybe sub-total, total, extended, or radical exenteration depending on the amount of orbital contents excised. Subtotal exenteration entails removal of only the anterior orbital tissue. In total exenteration, the orbital contents are removed in their entirety. In extended exenteration, removal of orbital bones is also performed. In radical exenteration, adjacent structures like the paranasal sinuses are also sacrificed.

Exenteration is generally considered to be a radical procedure that should be performed only when there is a valid indication (Table 12.2) [1620]. It is indicated in advanced primary orbital malignancies, eyelid tumors with orbital extension, conjunctival tumors with orbital extension, and intraocular tumors with orbital extension. Rarely, it may even be indicated in advanced benign tumors like neurofibromatosis or arteriovenous malformation with orbital deformity [19].
Table 12.2 Indications for exenteration

Advanced primary or recurrent orbital malignancies

Advanced primary or recurrent eyelid tumors with orbital extension

Advanced primary or recurrent conjunctival tumors with orbital extension

Advanced primary or recurrent intraocular tumors with orbital extension

Advanced benign tumors like neurofibromatosis with orbital deformity

\section{Preoperative Evaluation}

Preoperative evaluation of a patient with an ocular tumor includes documentation of symptoms, vision, pressure, slit lamp findings, and fundus findings. Additionally, transillumination test is performed for a suspected ciliary body tumor. The extent and nature of the tumor is assessed with the help of fundus images, ultrasound, fluorescein angiography (FA), and ocular coherence tomography (OCT). Examination of a child with a suspected intraocular tumor is always done under anesthesia, with complete evaluation including external photographs, RetCam images, and ultrasound performed in the same sitting. A large fundus diagram of the tumor replicating its extent in the eye with the measurements listed is used at the time of surgery.

For orbital tumors, careful inspection and palpation is necessary including evaluation of ocular motility, globe and lid position. Examination of the regional lymph nodes and other possible relevant details must be done. Radiological images of the orbit to study the tumor extent are obtained which must be available at the time of surgery. Malignancies with the potential for systemic spread must undergo a complete metastatic workup. In tumors which require adjunctive treatment with chemotherapy, radiotherapy or immunotherapy, a collaborative team consisting of medical and radiation oncology is essential. An incisional biopsy for the histopathologic diagnosis of the malignancy is recommended before proceeding with exenteration. 


\section{Technique of Enucleation (Video 12.1)}

It is pertinent that fundus examination be done by the operating surgeon at the time of surgery to identify and mark the correct eye. Enucleation can be performed under general anesthesia or local anesthesia with intravenous sedation. Even for cases done under general anesthesia, it is advised that retrobulbar injection of $2 \%$ lignocaine in combination with 1:100,000 epinephrine, mixed with $75 \%$ bupivacaine (1:1), be administered $(8-10 \mathrm{~mL})$.

After the patient is prepared and draped, a wire speculum is placed to exclude the eyelashes from the field. A lateral canthotomy is performed when additional space is required, as in an eye with retinoblastoma where the globe maybe enlarged $[2,21]$. A $360^{\circ}$ peritomy is performed using a blunt tipped Westcott scissors, cutting as close to the limbus as possible to preserve the conjunctiva (Fig. 12.1a). The underlying posterior tenon's fascia is undermined in all four quadrants between the recti muscles in a spreading action using a blunt tipped tenotomy scissors. The medial rectus muscle is hooked using a muscle hook and freed from the surrounding intermuscular septa by blunt dissection. The muscle is then identified, hooked and double-tagged, first with 6-0 silk suture $2 \mathrm{~mm}$ from the insertion and then with 6-0 vicryl suture $4 \mathrm{~mm}$ from the insertion (Fig. 12.1b, c) [2, 21]. 6-0 silk sutures serve as traction sutures while 6-0 vicryl sutures would later be used to suture the muscles through the implant or the conjunctiva (described later). The muscle is then transected between the two sutures. This is followed by similar tagging and transection of inferior rectus, lateral rectus, and superior rectus. Superior oblique and inferior
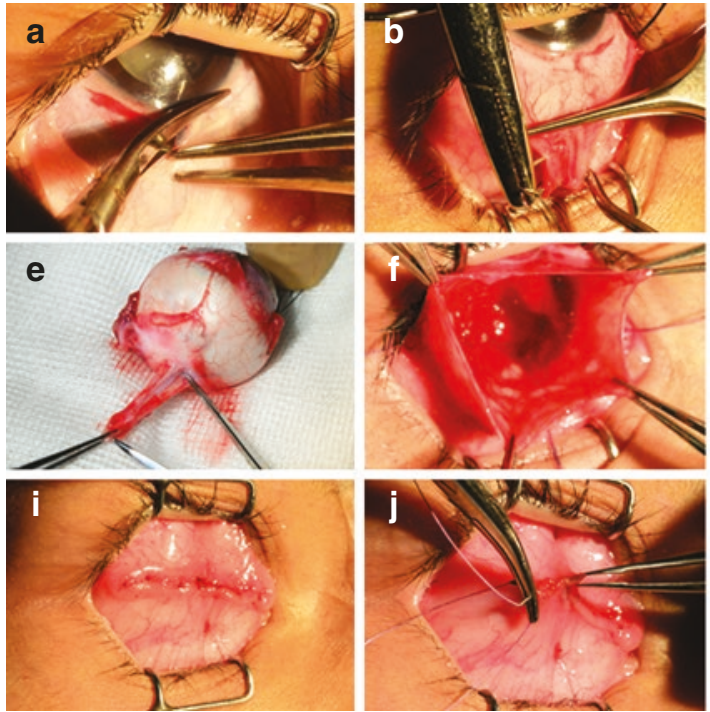

Fig. 12.1 Technique of enucleation in a 5-year-old male with right eye advanced retinoblastoma. (a) A $360^{\circ}$ peritomy is performed using a blunt-tipped Westcott scissors, cutting as close to the limbus as possible. After undermining the posterior tenon's fascia, each rectus is double tagged, (b) first with 6-0 silk suture $2 \mathrm{~mm}$ from the insertion and then with (c) 6-0 vicryl suture $4 \mathrm{~mm}$ from the insertion. After transecting the muscles, (d) the eyeball is prolapsed between the blades of the speculum, and the optic nerve divided just a little anterior to the superior orbital fissure. (e) The transected optic nerve length is
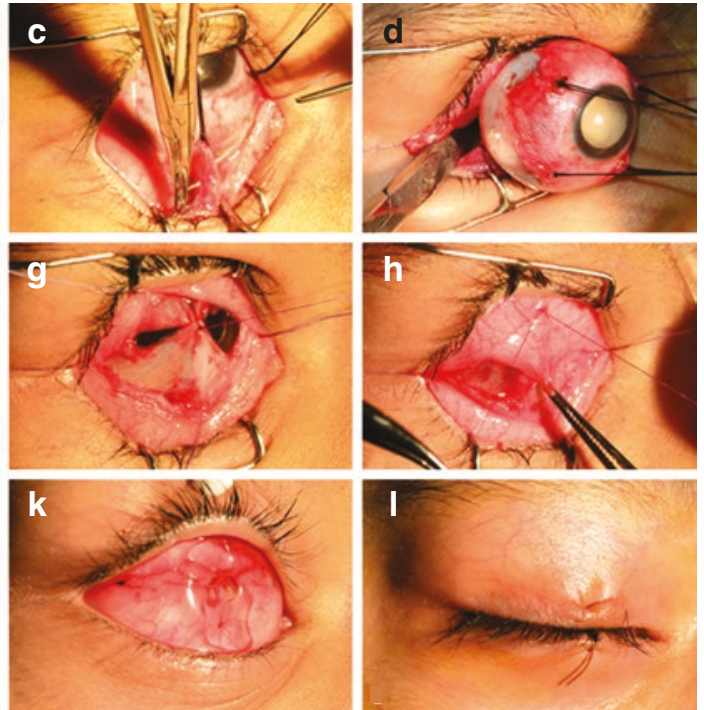

measured in all cases of retinoblastoma before the eyeball is submitted for histopathology. (f) Hemostasis is achieved and (g) the orbital implant placed within the posterior tenon's fascia, closing it with interrupted 6-0 vicryl suture. (h) Each rectus is sutured to the conjunctiva in its respective fornix by passing the tagged 6-0 vicryl suture through the conjunctiva. (i) Anterior tenon's closure is done with interrupted 6-0 vicryl suture and (j) conjunctival closure with a continuous key-suturing pattern. (k) An appropriatesized conformer is placed in the socket and (l) a temporary median tarsorrhaphy performed with 6-0 vicryl suture 
oblique muscles are transected and allowed to retract posteriorly. Each of the sutures is clamped with a bulldog clip.

A conjunctival relaxing incision is made for easy manipulation in large globes [21]. The eyeball is then prolapsed between the blades of the speculum (Fig. 12.1d). With a forward traction on the eyeball using the four silk sutures, a gently curved blunt-tipped tenotomy scissors is passed along the lateral wall and the optic nerve is strummed along its length. With one bold cut, the optic nerve is transected at the desired length. In retinoblastoma, the transection is performed just a little anterior to the superior orbital fissure, to gain a good length of the nerve $(15-20 \mathrm{~mm})$ and at the same time avoiding injury to the superior orbital fissure contents [2]. Adequate hemostasis is achieved using firm pressure for 5-10 $\mathrm{min}$. The enucleated globe is inspected thoroughly for any scleral thinning, perforation, extraocular tumor extension, and dilated vessels. The transected optic nerve length is measured (Fig. 12.1e), and the eyeball is then sent for histopathologic examination in $10 \%$ formalin.

An implant of adequate size and the surgeon's preferred material is placed posterior to posterior tenon's fascia (intraconal fat space). Posterior tenon's fascia is closed with interrupted $6-0$ vicryl sutures (Fig. 12.1f, g). The recti are then attached to the implant using the 6-0 vicryl sutures by passing the needles through posterior tenons including the implant wrapping material as anteriorly as possible. Alternately, the muscles can be sutured to the conjunctiva in their respective fornices by passing the needle through the conjunctiva (Fig. 12.1h). These are the myoconjunctival sutures and the technique is known as the myoconjunctival technique [21]. The advantage of this technique is that it allows the use of a non-integrable orbital implant (silicone or PMMA sphere) which offers a safe and costeffective alternative, with prosthesis motility comparable to biointegrable implants while minimizing the complications of implant extrusion and migration [21].

Following this, anterior tenon's is closed with interrupted 6-0 vicryl sutures (Fig. 12.1i). Conjunctival closure is done in a continuous key- suturing pattern with 6-0 vicryl suture (Fig. 12.1j). An appropriate sized conformer is placed in the socket (Fig. 12.1k) and a temporary median tarsorrhaphy performed with 6-0 vicryl suture (Fig. 12.11). The suture tarsorrhaphy is removed after 1 week, and a customized prosthesis can then be placed in the socket after 6 weeks [21].

\section{Choice of Implant}

Currently available implants can be categorized as:

1. Non-integrable: Implants that do not integrate with either the prosthesis or the orbital tissues. Examples for this include PMMA spheres, silicone spheres, and titanium spheres.

2. Semi-integrable: Implants that integrate with either the prosthesis or the orbital tissues. Allen's implant is an example for a semiintegrable implant.

3. Fully integrable: Implants that integrate with both the prosthesis and the orbital tissues. Cutler's implant is an example for a fully integrable implant.

4. Biointegrable: Implants that induce fibrovascularization within their substance and thus get integrated, for example, hydroxyapatite spheres.

5. Biogenic: Implants that are of biological origin, for example, dermis fat graft.

Non-integrated spherical implants by the conventional technique have a higher chance of migration, and also of poor implant and prosthesis motility. But when used in conjunction with the myoconjunctival technique, as described above, excellent static and dynamic cosmesis can be obtained [21]. Allen's implant and Cutler's implants are complicated by a high incidence of infection and extrusion [22]. Traditionally, dermis fat grafts are used in long-standing anophthalmic sockets with volume loss and surface loss [22].

Hydroxyapatite implants represent a new generation of integrated spheres that allow fibrovascular growth, which theoretically reduces the chances of migration, infection, and extrusion 
[23-25]. Also, these biointegrated implants with pegging achieve satisfactory excellent motility [23-25]. Porous polyethylene offers a good alternative to hydroxyapatite implants, as they cause less irritation to the conjunctiva owing to their smoother surface [22]. Porous implants, although in wide use, are known to cause certain complications. These include conjunctival irritation, discharge, pyogenic granuloma formation, implant exposure, scarring, implant infection, and persistent discomfort [22-25]. These implants are also not suitable in immunocompromised individuals, diabetics, or in those who have had or require periorbital radiation therapy [22]. The choice of implant used is left to the surgeon's discretion.

\section{Technique of Exenteration (Video 12.2)}

Exenteration can be performed by either an eyelid-sacrificing or eyelid-sparing technique. With the eyelid-sacrificing method, the orbital cavity has to be lined by temporalis muscle, forehead flaps or grafts. Some surgeons advocate spontaneous granulation, and this offers comparable cosmetic results. However, healing by granulation takes longer and requires intensive postoperative care.

To circumvent this problem, an eyelid-sparing technique maybe used when possible. This method, popularized by Coston and Small, is a modification of the total exenteration technique which spares parts of both the eyelids, with transverse blepharorrhaphy to cover the orbit, thus ensuring better cosmesis and early rehabilitation [17]. In addition, sparing of the orbicularis muscle provides an excellent vascular supply to the skin flap, enabling early wound healing $[17,20]$.

Assistance from an otorhinolayrngologist or a neurosurgeon may be necessary in patients with extensive maxillary sinus involvement or roof erosion by the orbital tumor. The correct eye is identified and marked. Exenteration is always performed under general anesthesia. After the patient has been prepped and draped, the incision marking is placed $2 \mathrm{~mm}$ behind the lash line, joining them at the medial and lateral commissures (Fig. 12.2a) [17]. Three double-armed trac- tion sutures with 4-0 silk are placed through the upper and lower tarsi to provide traction on the orbital contents. The incision is then made with a radiofrequency probe along the skin marking (Fig. 12.2b). Dissection is carried in the preseptal plane which decreases the likelihood of perforating the orbital septum, which is especially important in tumors present in the anterior orbit (Fig. 12.2c) [17]. Also, it leaves the orbicularis muscle intact, which provides a source for vascular supply to the skin flap.

Dissection is done till the orbital rim is reached, and the periosteum just outside the arcus marginalis is incised all around (Fig. 12.2d). Periosteal elevators are used to dissect the periosteum off the bony orbit, beginning at the incised margin along the orbital rim and continuing all the way back to the orbital apex (Fig. 12.2e). Most of the periosteum is loosely adherent to the underlying bone, with tight adhesions seen at certain anatomic locations, including the bony sutures and orbital fissures, as also in areas where the tumor has spread to the periosteum. At these points, gentle dissection is done to prevent any rip in the periosteum. Along the superior rim, dissection is gently carried around the supraorbital notch. The supraorbital and supratrochlear neurovascular bundle are identified and cauterized. Subperiosteal dissection is then carried from anterior to posterior lacrimal crest and beyond. Dissection is performed carefully along the medial wall, so as to not fracture the thin lamina papyracea. After negotiating the frontozygomatic suture with gentle dissection, zygomaticofacial and zygomaticotemporal neurovascular bundles are identified and cauterized. Dissection should be performed carefully along the orbital floor, so as to not fracture the thin bone and create a communication with the maxillary sinus. The sac is approached by dissecting medial to it and dividing the common canaliculus and orbicularis attachments. It is dissected from the lacrimal sac fossa and divided from the nasolacrimal duct with cautery. The exposed nasolacrimal duct is obliterated by cautery to reduce the risk of postoperative fistula formation (Fig. 12.2f).

Further deeper along the medial wall, anterior and posterior ethmoidal vessels are identified and 

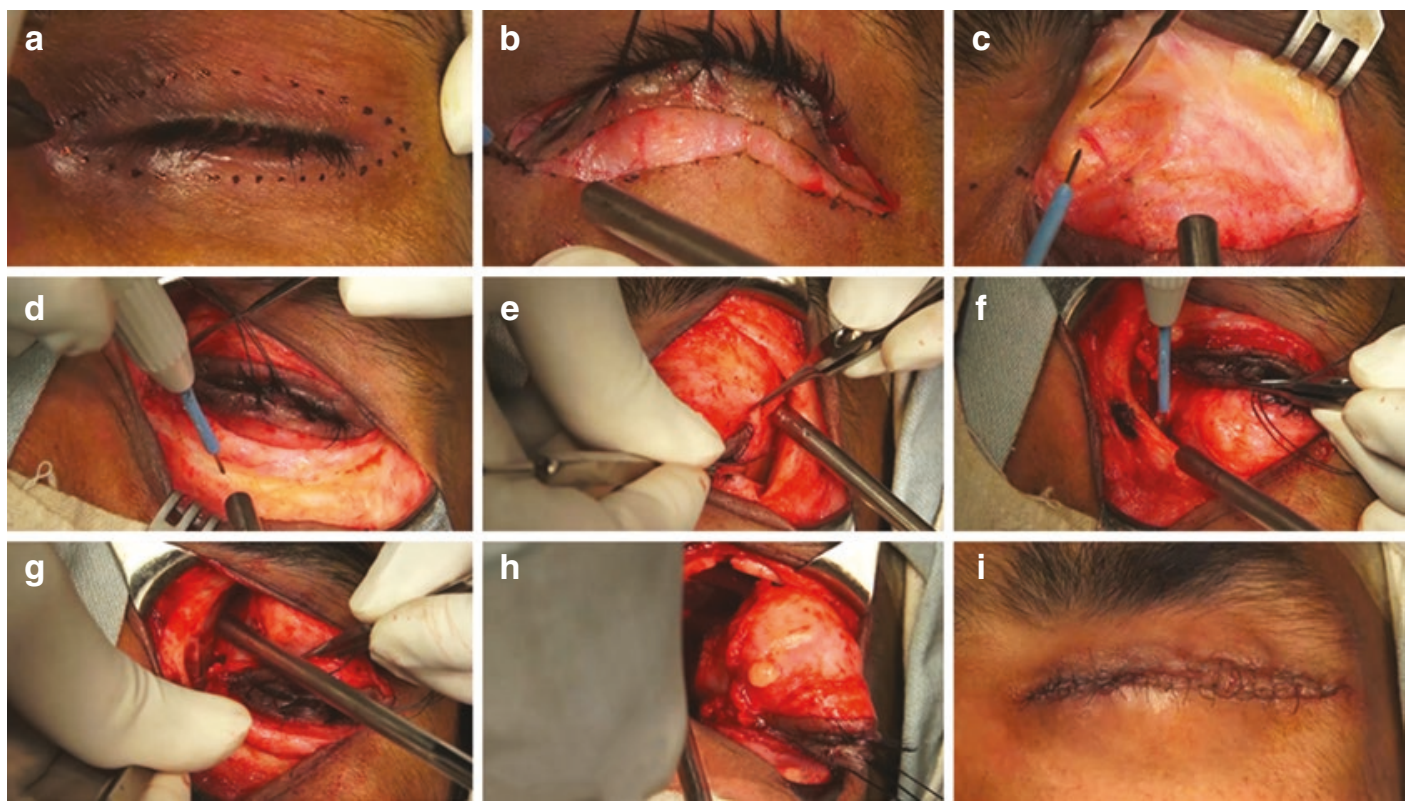

Fig. 12.2 Eyelid-sparing orbital exenteration technique in a 49-year-old male with the left orbital extension of conjunctival squamous cell carcinoma. (a) An incision marking is placed $2 \mathrm{~mm}$ behind the lash line in both the upper and lower eyelids, joining them at the medial and lateral commissures. (b) The incision is made with a radiofrequency probe along the skin marking. (c) Dissection is carried in the preseptal plane till the orbital rim is reached. (d) The periosteum just outside the arcus marginalis is incised all around. (e) A periosteal elevator is used to dissect the periosteum off the bony orbit, begin-

cauterized (Fig. 12.2g). In the inferotemporal orbit, the inferior orbital fissure is encountered and penetrating vessels divided with cautery. Next, the infraorbital nerve is identified and cauterized. Ensure that the periosteum has been dissected to the apex, by passing a lid speculum all around in the orbit. A pair of curved enucleation scissors is then introduced into the posterior orbit. The optic nerve, superior orbital fissure contents, and posterior orbital tissues are divided (Fig. 12.2h). Hemostasis can be obtained with ice-cold wet gauze, pressure, and/or cautery. If necessary, additional hemostasis is achieved with surgicel or bone wax. The socket is carefully inspected for any residual tumor. The adequacy of resection may be judged with the aid of frozensection control. The resection of additional orbital apical tissue may be done as deemed necessary. The eyelid flaps are reapproximated in ning at the incised margin along the orbital rim and continuing all the way back to the orbital apex. (f) After dividing the nasolacrimal duct, the exposed duct is obliterated by cautery. (g) Along the deeper medial wall, anterior and posterior ethmoidal vessels are identified and cauterized. (h) The orbital tissue is held in forward traction while dividing the posterior structures. (i) After adequate hemostasis, the eyelid flaps are reapproximated in two layers, with the orbicularis closure using 4-0 vicryl and skin closure using 6-0 silk suture

two layers, with orbicularis closed using 4-0 vicryl, and the skin using 6-0 silk suture (Fig. 12.2i) [17]. Aspiration of the orbital cavity is done at the end of the procedure using a $10 \mathrm{cc}$ syringe which yields some amount of blood and serum, and provides a concave lid flap. The wound is then patched using an antibiotic ointment.

Following surgery, aspiration of the socket for any blood or serum is done every day using a $10 \mathrm{cc}$ syringe till a dry aspiration is obtained. The socket usually heals quickly over the course of 3-6 weeks and is ready for a prosthesis at the end of 6-8 weeks. Eyelid-sparing orbital exenteration enables early wound healing and cosmetic rehabilitation thus minimizing patient morbidity.

For lid-sacrificing exenteration, the incision marking is placed along the orbital rim. Dissection is then carried till the periosteum. 
The periosteum just outside the arcus marginalis is incised, and the rest of the steps are similar as above. After excision of the orbital contents, the orbital cavity is left open to granulate in which case epithelialization generally occurs over 3-4 months, or reconstruction using split skin lining or skin flaps is done.

Following enucleation and orbital exenteration, the challenge lies in providing a good cosmesis. Once complete healing of the wound has occurred, a customized ocular prosthesis in an enucleated socket can be placed in 6 weeks. A good surgical technique is essential to provide a concave, smooth, and stable skin cover, over which an orbital prosthesis can be placed $[17,20]$.

In conclusion, enucleation and exenteration in ocular oncology are mainly performed in advanced malignant tumors. These procedures can be life-saving and are used as a part of multimodal treatment in achieving complete local remission. The cosmetic disfigurement caused by these radical procedures must be rehabilitated by a well-matched prosthesis.

\section{References}

1. Shields CL, Shields JA. Retinoblastoma management: advances in enucleation, intravenous chemoreduction, and intra-arterial chemotherapy. Curr Opin Ophthalmol. 2010;21(3):203-12.

2. Honavar SG, Singh AD. Management of advanced retinoblastoma. Ophthalmol Clin N Am. 2005;18:65-73.

3. Murthy R, Honavar SG, Naik MN, Reddy VA. Retinoblastoma. In: Dutta LC, editor. Modern ophthalmology. New Delhi: Jaypee Brothers; 2004. p. 849-59.

4. Honavar SG. Orbital retinoblastoma. In: Singh AD, Murphee LA, Damato BE, editors. Clinical ophthalmic oncology-retinoblastoma. 2nd ed. New York: Springer; 2015.

5. Shields CL, Shields JA. Ocular melanoma: relatively rare but requiring respect. Clin Dermatol. 2009;27(1):122-33

6. Shields J, Shields C. Posterior uveal melanoma: clinical features. In: Shields J, Shields C, editors. Atlas of intraocular tumors. 3rd ed. Philadelphia: Lippincott, Wolters Kluwer; 2016. p. 45-59.

7. Lingam G. Options for management of intra ocular tumors. Indian J Ophthalmol. 2015;63(3):204-10.
8. Rini FJ, Jakobiec FA, Hornblass A, Beckerman BL, Anderson RL. The treatment of advanced choroidal melanoma with massive orbital extension. Am J Ophthalmol. 1987;104:634-40.

9. Gündüz K, Shields CL, Shields JA, Cater J, Brady L. Plaque radiotherapy for management of ciliary body and choroidal melanoma with extraocular extension. Am J Ophthalmol. 2000 Jul;130(1):97-102.

10. Popovic M, Ahmed II, DiGiovanni J, Shields CL. Radiotherapeutic and surgical management of iris melanoma: a review. Surv Ophthalmol. 2017;62:30211. pii:S0039-6257(16)30152-7.

11. Shields CL, Shah SU, Bianciotto CG, et al. Iris melanoma management with iodine-125 plaque radiotherapy in 144 patients: impact of melanoma-related glaucoma on outcomes. Ophthalmology. 2013;120:55e61.

12. Kaliki S, Shields CL, Eagle RC Jr, et al. Ciliary body medulloepithelioma: analysis of 41 cases. Ophthalmology. 2013;120(12):2552-9.

13. Shields JA, Shields CL, Sivalingam V. Decreasing frequency of enucleation in patients with retinoblastoma. Am J Ophthalmol. 1989;108:185-8. 32.

14. Ferris FL 3rd, Chew EY. A new era for the treatment of retinoblastoma. Arch Ophthalmol. 1996;114:1412.

15. Epstein JA, Shields CL, Shields JA. Trends in the management of retinoblastoma: evaluation of 1,196 consecutive eyes during 1974 to 2001. J Pediatr Ophthalmol Strabismus. 2003;40:196-203.

16. Kennedy RE. Indications and surgical techniques for orbital exenteration. Ophthalmology. 1979;86(5):967-73.

17. Coston TO, Small RG. Orbital exenteration-simplified. Trans Am Ophthalmol Soc. 1981;79:136-52.

18. Rathbun JE, Beard C, Quickert MH. Evaluation of 48 cases of orbital exenteration. Am J Ophthalmol. 1971;30;72(1):191-9.

19. Levin PS, Dutton JJ. A 20-year series of orbital exenteration. Am J Ophthalmol. 1991;112(5):496-501.

20. Shields JA, Shields CL, Demirci H, Honavar SG, Singh AD. Experience with eyelid-sparing orbital exenteration: the 2000 Tullos O. Coston lecture. Ophthal Plast Reconstr Surg. 2001;17(5):355-61.

21. Shome D, Honavar SG, Raizada K, Raizada D. Implant and prosthesis movement after enucleation: a randomized controlled trial. Ophthalmology. 2010;117(8):1638-44.

22. Leatherbarrow B. Enucleation and evisceration. In: Leatherbarrow B, editors. Oculoplastic surgery. 2nd ed. Informa Healthcare; London, 2011. p. 479-98.

23. Kim YD, Goldberg RA, Shorr N, Steinsapir KD. Management of exposed hydroxyapatite orbital implants. Ophthalmology. 1994;101:1709-15.

24. Shields JA, Shields CL, De Potter P. Hydroxyapatite orbital implant after enucleation-experience with 200 cases. Mayo Clin Proc. 1993;68:1191-5.

25. Kaltreider SA, Newman SA. Prevention and management of complications associated with the hydroxyapatite implant. Ophthal Plast Reconstr Surg. 1996;12:18-31. 
Open Access This chapter is licensed under the terms of the Creative Commons Attribution 4.0 International License (http://creativecommons.org/licenses/by/4.0/), which permits use, sharing, adaptation, distribution and reproduction in any medium or format, as long as you give appropriate credit to the original author(s) and the source, provide a link to the Creative Commons license and indicate if changes were made.

The images or other third party material in this chapter are included in the chapter's Creative Commons license, unless indicated otherwise in a credit line to the material. If material is not included in the chapter's Creative Commons license and your intended use is not permitted by statutory regulation or exceeds the permitted use, you will need to obtain permission directly from the copyright holder. 